\title{
EFPT-OIDS: Evaluation Framework for a Pre-processing Techniques of Automatic Optho- Imaging Diagnosis and Detection System
}

\author{
Sobia Naz ${ }^{1}$ \\ Research Scholar \\ Department of Electronics and \\ Communications Engineering \\ PES College of Engineering \\ Mandya, Karnataka, India
}

\author{
Dr.Radha Krishna Rao K.A ${ }^{2}$ \\ Professor and Head \\ Department of Electronics and \\ Communications Engineering \\ PES College of Engineering \\ Mandya, Karnataka, India
}

\author{
Dr. Shreekanth $\mathrm{T}^{3}$ \\ Project Manager \\ L\&T Technology Services \\ Mysore, India
}

\begin{abstract}
The modalities of FUNDUS images and the availability of public domain data sets provides a starting point in designing an ecosystem for developing an automatic detection of degenerative early-stage Glaucoma and Diabetic Retinopathy, and other eye-related diseases. The existing techniques for these operations lack flexibility and robustness in their design implementation and are limited to only certain preprocessing requirements. However, the existing methods are useful but provide lower performance when the FUNDUS image quality degrades due to misalignment of lens opening in camera and poor functioning of visual sensors. This paper presents a unified framework that mechanizes different preprocessing techniques to benefit the Optho-imaging diagnosis and disease detection process. The proposed framework facilitates on-demand data treatment operations that include image interpolation, brightness adjustment, illumination correction, and noise reduction. The proposed techniques for FUNDUS image enhancement provide better PSNR and SSIM-performance metrics for image quality than existing popular image enhancement techniques when tested on two standard publicly available datasets. The contribution of the proposed framework is that it offers flexible and effective mechanisms that meet dynamic preprocessing operations on an on-demand basis to prepare better data representation for building machine learning models. The framework can also be used in real-time for eye disease diagnosis by an ophthalmologist.
\end{abstract}

Keywords-Pre-processing; FUNDUS image; glaucoma; diabetic retinopathy; interpolation; image enhancement

\section{INTRODUCTION}

The multidomain research modality is gaining popularity with the ever-increasing availability of the dataset, computing platform, and ecosystem, along with the advancement of the technologies like artificial intelligence (AI) and machine learning (ML) [1]. Many of the system processes aim to obtain complete automation in the enterprise's applications. The health care industry is one of the fastest adopting sectors after defense and another industrial automation [2]. The research statistics reveal that the stake of $\mathrm{AI}$ in medical imaging is approximately USD 264 billion by 2026 [3]. This paper focuses on medical imaging, namely, Fundus Imagining for the specialized healthcare section called ophthalmology, which deals with diagnosing and treating eye-related disease
Glaucoma from preprocessing perspectives. It is essential to know the modality of the Fundus image capturing aspects to understand the inclusion of the noises or redundancies that require preprocessing operation for the human vision system (HVS). The ophthalmic photographers capture the retina image using a specially designed fundus camera [4]. Fundus images are quite popular; the ophthalmologists found it suitable to diagnose Glaucoma or diabetic retinopathy [5]. The fundus images produce shallow and non-uniform contrast conditions between the retinal structural elements and the background and due to which the ophthalmologist faces difficulty to analyze it. However, the clarity is found by practicing an injective of the contrasting chemical during the fundus angiogram, but it sometimes affects the patient's health conditions [6]. The image enhancements techniques provide an alternate noninvasive way to enhance the fundus image quality for the HVS.

Further, with the increasing number of patients related to eye diseases and the lack of adequate ophthalmologist experts, investment is needed to build an automated system that can diagnose eye diseases using AI and ML. There is a critical requirement of preprocessing on Fundus images to obtain a higher accuracy for segmentation and feature extraction for learning models. The essential and effective preprocessing techniques include 1) fundus image enhancement for contrast and brightness adjustment 2) Interpolation for uniform dataset images 3) Region of Interest (ROI) localization for vessel removal by cropping for higher accuracy by the learning model. The existing literature is rich in treating medical image quality but, with a limited scope, does not consider all the essential preprocessing techniques in a single framework. Also, the existing techniques implemented using recursive operation suffers from substantial computational complexity that hinders its applicability in the real-time scenario. In this regard, the proposed work suggests a unified preprocessing framework consisting of different types of enhancement and image correction techniques that can meet the dynamic preprocessing requirement without depending on the requirement of an external source. The current research work also proposes a image enhancement techniques based on the convolution operation to filter the noise in frequency domain 
and enhance the contrast without affecting the diagnostic quality of the image. The rest of the sections of this paper includes: related work in Section II, research problem in Section III description of the dataset is presented in Section IV. The modeling of the proposed preprocessing framework is discussed in Section V. Result analysis for the proposed preprocessing approaches is given in Section VI, and the overall contribution of this paper is summarized in Section VII.

\section{RELATED WORK}

Over the past few years, several techniques have been introduced to enhance fundus images before diagnosing optic disc for inspecting the condition of the diseases or Glaucoma that affect the eye. A work carried out by Sudeshna, and Santi [7] presents an automatic model to identify retinal lesions, where the curvelet transform technique is used for the edge enhancement and dark and brighter areas of retinal lesions are optimized using a bandpass filter. However, this approach requires a higher computational time. Image contrast enhancement using adaptive histogram equalization (AHE) was quite popular; the study by Bharkad [8] has applied AHE on the green component of the retinal to enhance the contrast for segmenting optic disk. A similar approach has been considered in the study of Jebaseeli et al. [9] to enhance the green component towards improving the contrast of the blood vessels. The work of Bhatt et al. [10] considers AHE on the red component of the retinal image for normalizing contrast. On the other hand, the median filtering approach is considered for the smoothing optic disc regions. However, AHE-based contrast enhancement provides improvements at the cost of reduced gray level, which may associate with loss of image intelligibility. In the study of Sahu et al. [11], the authors have demonstrated that CLAHE based preprocessing is appropriate for the refining fundus image quality. The work done by Rahim et al. [12] suggested multiple approaches to enhancing the fundus image towards the recognition of blood vessels for diabetic retinopathy using AHE, CLAHE, and Mahalanobis Distance techniques applied over the green channel of the fundus image. However, the Mahalanobis Distance method shows effective performance compared to the other two methods. In the study of Elloumi et al. [13], the authors presented a pipeline for image enhancement for the fundus image captured from the smartphone. They suggested that image enhancement can be better achieved by adopting the CLAHE and Butterworth filtering approach to reduce highfrequency noise present in the smartphone-captured fundus image. However, this approach may suffer from some real problem that has not been explored and addressed in this work. Bala et al. [14] adopted a joint approach of AHE and curvelet features for denoising retinal images. AHE is considered for optimizing ringing in this approach, and specular noises and curvelet features are considered for edgepreserving during denoising operation. The use of a quadratic filter can also be seen in the study of Hari et al. [15] to localize diabetic retinopathy. Abdallah et al. [16] presented their work to address the excessive smoothing issues caused by linear filters and conducted a performance assessment of different diffusion filters to improve contrast and sharpness of the fundus image for disease analysis. A de-hazing technique is adopted in Vinodhini et al. [17] to correct non-uniform illumination in fundus image intensity, and contrast enhancement is performed using CLAHE followed by an adaptive median filter. Bhardwaj et al. [18] evaluated mathematical morphology for optic disc segmentation and blood vessel extraction. The work carried out by Hassan and Hassanien [19] developed an automated system to extract the retinal vasculature from the fundus images using preprocessing and segmentation mechanisms. The preprocessing operation over the fundus image is carried in multiple steps, where the first step is the fundus image resizing. The global mean value is computed for the brightness pixel, and further windowing-mean operation is carried to compute an enhanced image. Apart from AHE, CLAHE, Morphology, median filtering, and curvelet transform, Retinex-based preprocessing schemes have also been introduced to enhance the contrast of the Fundus or retinex images. The work of Sadia et al. [20] and Mahmood et al. [21] demonstrated the effectiveness of multi-scale retinex based preprocessing techniques for high-quality enhancement by eliminating uneven illumination in the image intensity. The adoption of hybrid approaches is also seen in some studies. A recent work by Sathananthavathi and Indumathi [22] uses a particle swarm optimization approach with gamma correction to improve and achieve optimal enhancement on the fundus image. Alwazzan et al. [23] used wiener filters with CLAHE to enhance the color fundus image. Bataineh and Almotairi [24] adopted bilateral filters to highlight the visual feature of the fundus image. However, all these techniques have significant dependency on large computational resource requirements due to their implementation strategy's recursive nature. Irrespective of having many suitable techniques, the ophthalmologist and machine learning modelers for Opthoimaging requires an integrated framework that can be used for both diagnostic purposes and data perpetrator.

\section{RESEARCH PROBLEM}

Despite several research efforts, the existing schemes for the preprocessing fundus image still have substantial issues that need to be improvised with a unique implementation strategy. Based on review analysis, it has been found that most of the existing researchers have adopted common techniques such as Gamma correction Adaptive Histogram, CLAHE, and Retinex, for fundus image preprocessing. However, these techniques offer better results, but at the same time, they may suffer a lack of flexibility and performance challenges when applied on different datasets as the existing techniques are particular to their objectives. Along with the issue of robustness and flexibility, the existing schemes suffer from the overhead of computational complexity and take longer runtime. Therefore, it is necessary to design an integrated framework to meet the preprocessing requirements by solving dynamic problems in order to achieve flexible, computationally efficient, and effective fundus image enhancement. The model must be robust enough to complement ophthalmologist diagnosis needs and be an easy tool to generate data for automated ophthalmological disease model learning. 


\section{DATASET Description}

The proposed work for designing an evaluation framework, EFPT- OIDS, provides an ecosystem to visualize the raw images from the different datasets for the ophthalmologist, Optho-data scientist, and analytics. The EFPT- OIDS is evaluated on two publicly accessible standard datasets $(\mathrm{Ds})=\{$ CHASEDB 1 , DRIONS-DB $\}$. The summary of these datasets are highlighted in Table I:

\section{A. CHASEDBI}

The CHASEDB1 is a reference database of retinal blood vessel segmentation provided by Child Health and Study England (CHASE). The database was obtained from a health survey conducted in London over 200 primary schools, and 20 fundus images of 14 children were captured at 30o Field of View with a resolution of $1280 \times 960$ to prepare the CHASE dataset. The data set is publicly available to facilitate researchers for experimental purposes. The fundus images in this database are associated with uneven illumination background and low contrast of blood vessels. The 28 images that make up the CHASEDB1 database are divided into a testing set (20 Fundus images) and a training set ( 8 Fundus images).

\section{B. DRIONS-DB}

DRIONS database is subjected to digital fundus image for optic nerve segmentation and open to public access for research purposes. The dataset was prepared using images belonging to the patient (male $46.2 \%$ and female 53.8\%) subjected to Caucasian ethnicity. About $76.9 \%$ of patients were suffering from eye hypertension, and $23.1 \%$ of patients were suffering from chronic simple Glaucoma disease. The dataset contains 110 color images having a resolution of $600 \mathrm{x}$ 400 , and two experts manually segmented the optic disc.

TABLE I. DESCRIPTION OF DATABASES

\begin{tabular}{|l|l|l|l|l|}
\hline SI. No & Databases & Resolution & Number & Application \\
\hline 1 & CHASEDB1 & $1280 \times 960$ & 28 & $\begin{array}{l}\text { Blood vessels } \\
\text { segmentation }\end{array}$ \\
\hline 2 & DRIONS-DB & $600 \times 400$ & 110 & $\begin{array}{l}\text { Optic nerve } \\
\text { segmentation }\end{array}$ \\
\hline
\end{tabular}

\section{DESIGN OF PROPOSED EFPT-GDS}

The fundus images contain an uneven illumination due to various real-time constraints like lightning condition, misalignments of retinal and camera focus, and faults in the camera that degrades fundus images. These images require preprocessing towards enhancement from both HVS and computer vision system (CVS) analysis viewpoints. Also, exploration of the FI dataset and its Interpolation is the requirement for preprocessing. The core component of the framework includes i) FI visualization, ii) FI Interpolation, iii) Region of Interest extraction and iv) Existing and Proposed enhancement.

\section{A. Fundus Image Visualization}

The framework creates a pointer for the location (Dp) of the dataset. Further, the encoded image representation (Ie) gets concatenated as $(\mathrm{Dp} \cup \mathrm{Ie})(\mathrm{Dn}$, where, $\mathrm{Dn}$ is the locator $\forall \mathrm{Ie} \in$ $\mathrm{Dp}$, the list of $\forall$ Dn gets updated into a structure (Is) as $\{(\mathrm{Ie}) \mathrm{i}$,
Ts, M , where $\mathrm{i}=$ total number of the $\mathrm{Ie} \in \mathrm{Dp}$, Ts is time stamping. $M$ is the size of Ie in bytes. For $\forall$ Ie $\in$ Is, perform sampling and quantization to get the digitized equivalent matrix as I[]m,n,d, where $\mathrm{m}$ is several rows, $\mathrm{n}$ is the number of columns, and $\mathrm{d}$ is the number of dimensions, for original fundus image, $d=3$ as $[R, G, B]$ color space. Finally, the framework displays Ie $\in$ Is: Dp. Fig. 1 illustrates 'k=5' number of Ie $\in$ Is: D The Ophthalmologist can use the FI visualization tool to diagnose eye diseases, whereas this facility of the framework is helpful for the modeler to data exploration.
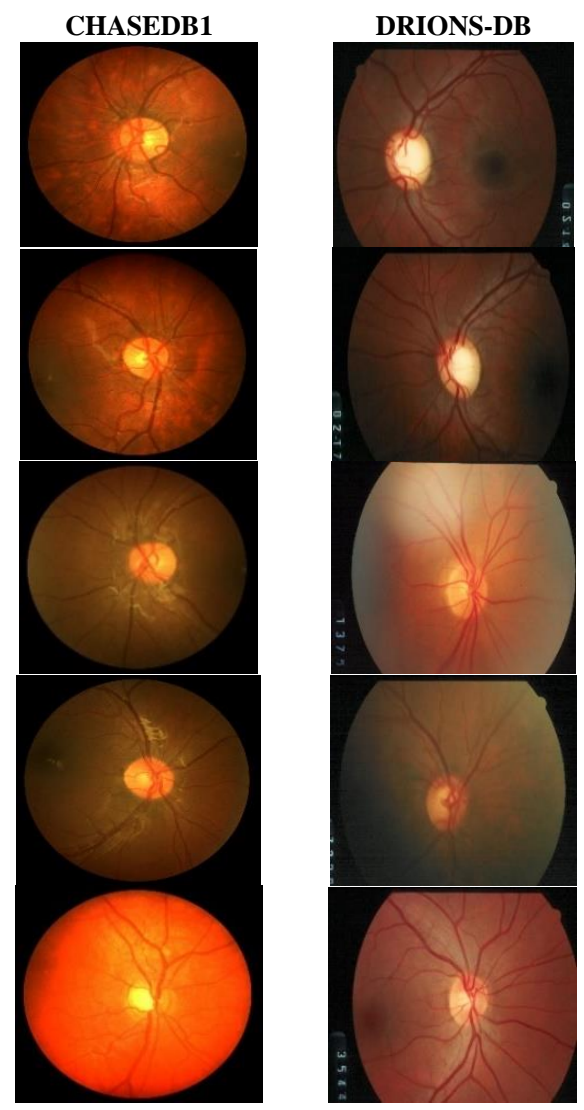

Fig. 1. Visualization 'k' Fundus Images from a Chosen Dataset CHASEDB1 and DRIONS-DB (Ie $\in$ Is: Dp.

\section{B. Fundus Image Interpolation}

The ophthalmologists require an eye image with highly distinct retina layers to diagnose glaucoma [25]. Fundus photography aims to provide such imaging methodologies in their imaging process [26]. Though the ophthalmic photographer adopts various field strategies to achieve the perceived enhanced sharpness in the Fundus imagining, it requires additional processing by computing image processing. The requirement of the down sampling and up a sampling of fundus images (FI) arises while the development of computing models for the automatic detection of Glaucoma (ADG)[27] and other eye-related diseases. One such requirement includes maintaining the equal size of the fundus images from different datasets for validating the developed model of automatic disease detection (ADD) on the different datasets as different datasets maintain different sizes of the FI [28-29]. The lowest resolution of the $\forall$ FIo $\in \mathrm{Dp}$ is set to a threshold $=\{$ Rth, Cth $\}$ of the target size of scaling to obtain an 
interpolated or scaled fundus image (FIs). The framework includes a tool for FI interpolation using the nearest neighbours as in algorithm 1 . The algorithm takes the input of original funds image-(FIo), and after processing, it provides interpolated enhanced fundus image-(FIs). The algorithm computes the size of FIo as row size (nR) and column size $(\mathrm{nC})$. The system then initializes the variables Rth (Target image row size) and Cth (target image column size) as a threshold to obtain an interpolated fundus image.

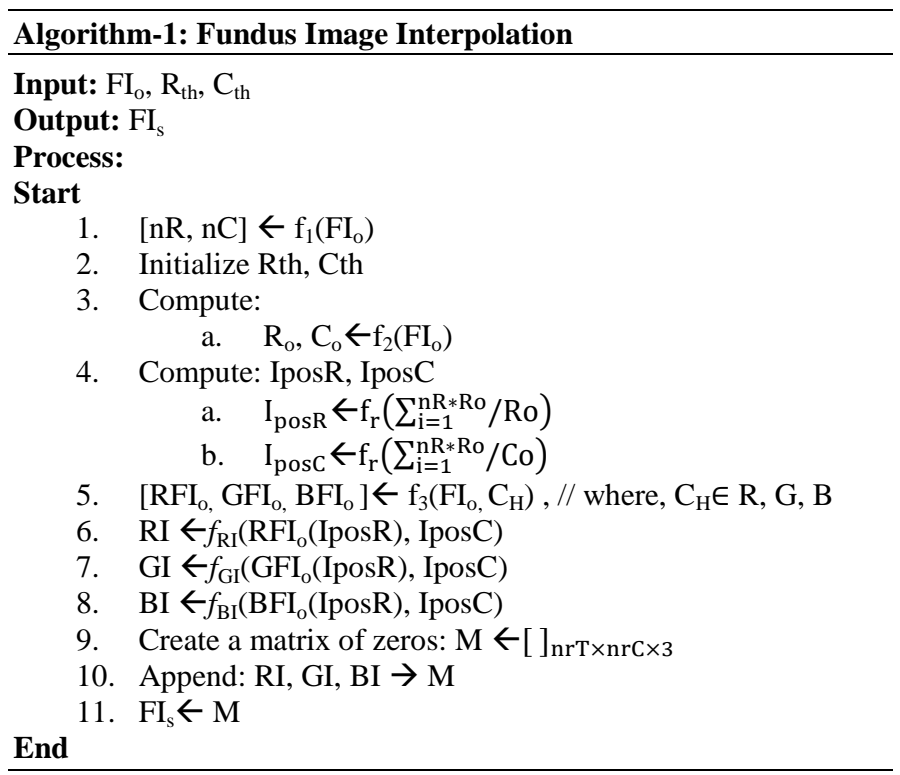

In the next step, the system computes the ratio $\left(\mathrm{R}_{0}, \mathrm{C}_{0}\right)$ of target image size and size of $\mathrm{FI}_{\mathrm{o}}$ using explicit function $\mathrm{f}_{2}$ described in equation 1 and equation 2 .

$$
\begin{aligned}
& \mathrm{R}_{0}=\frac{R_{t h}}{\mathrm{nR}} \\
& \mathrm{C}_{0}=\frac{C_{t h}}{\mathrm{nC}}
\end{aligned}
$$

Further, a function $\mathrm{f}_{\mathrm{r}}(\mathrm{)})$ is applied over the interpolated position to compute normalized the row-wise pixel position$\left(I_{\text {posR }}\right)$ and column-wise pixel position- $\left(I_{\text {posC }}\right)$ considering Ro, $\mathrm{Co}$, and the size of $\mathrm{FI}_{\mathrm{o}}$ as given in equation 3 and equation 4 .

$$
\begin{aligned}
& \mathrm{I}_{\text {posR }}=\mathrm{f}_{\mathrm{r}}\left(\sum_{\mathrm{i}=1}^{\mathrm{nR} * \mathrm{Ro}} / \mathrm{Ro}\right) \\
& \mathrm{I}_{\text {posC }}=\mathrm{f}_{\mathrm{r}}\left(\sum_{\mathrm{i}=1}^{\mathrm{nR} * \mathrm{Ro}} / \mathrm{Co}\right)
\end{aligned}
$$

Where $\operatorname{fr}()$ denotes rounding to the nearest integer. In the next step of the algorithm, the system performs row-wise and column-wise Interpolation considering red-( $\left.\mathrm{RFI}_{\mathrm{o}}\right)$, green $\left(\mathrm{GFI}_{\mathrm{o}}\right)$, and blue- $\left(\mathrm{BFI}_{\mathrm{o}}\right)$ components extracted from the FIo. Further, Interpolation for all three components, such as red component-(RI), green component-(GI), and blue component(BI) is computed. In the next step, the algorithm creates a matrix-(M) of zeros with size $\mathrm{R}_{\mathrm{th}}$ and $\mathrm{C}_{\mathrm{th}}$. Further, it performs an appending operation that resamples the interpolated RGB component's pixel value in matrix $\mathrm{M}$ to generate an enhanced interpolated image (FIs) outcome. In Fig. 2, the rescaling of the fundus input image using nearest-neighbour Interpolation is carried out, and the output fundus images are constructed with the specified dimension without losing details of the fundus image.

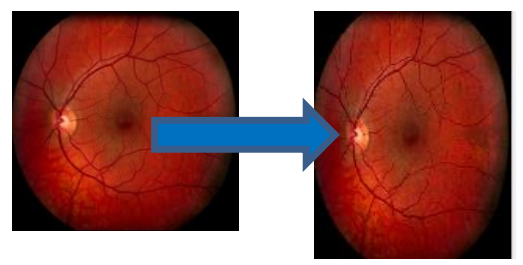

$$
\begin{array}{cc}
\text { Input Fundus Image } & \text { Rescaled Fundus Image } \\
(512 \times 576) & (700 \times 500)
\end{array}
$$

Fig. 2. FI Interpolation using Nearest Neighborhood Interpolation Method.

\section{Region of Interest Extraction}

Since the input FI in the dataset comes with different resolutions and fields of views (FOVs). To extract the region of interest and eliminate the background from the input FI, the proposed framework adopts an adaptive cropping method that creates an interactive rectangular mask to cover the RoI region of the input FI. The tool comes with a flexible approach to operate at the ease of the user on the rectangular mask. Fig. 3 illustrates the RoI extraction from FI.

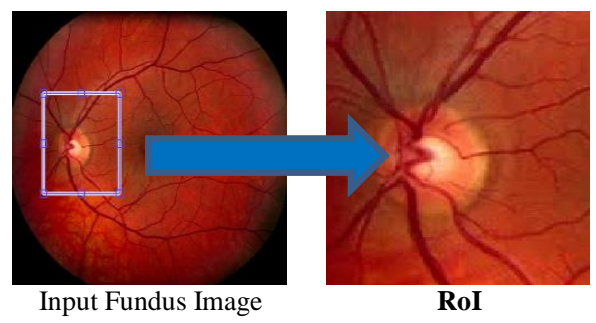

Fig. 3. RoI Extraction using user Interactive Cropping Method.

In Fig. 3, the left-hand side image is the input or target image, and the right-hand side is the RoI extracted from the input fundus image using a rectangular mask provided by the cropping tool.

\section{Gamma Correction for Fundus Image Enhancement}

The operator gamma ( $\boldsymbol{\gamma})$ performs encoding of the luminance of the input fundus images. The representation of this correction is popular by the name Gamma-Correction, and it is characterized as in eq.5.

$\mathrm{I}_{\text {output }}=\mathrm{C} \times\left(\mathrm{I}_{\text {input }}\right)^{\gamma}$

In this method, the input image ( $\left.\mathrm{I}_{\text {input }}\right)$ is mapped to the $\mathrm{I}_{\text {output }}$ by applying operator, $\mathrm{C}$ as a constant and $\gamma$ as encoding and decoding factor, which ranges as $0 \leq \gamma \leq 1$ for a compressed domain but in the process of the image enhancement, an optimal value of it needs to be arrived based on the best PSNR.

In a particular context where only the gamma correction is suitable, the framework provisions manual adjustment of $\gamma$ to arrive at a better visual perception from the HVS viewpoint. However, it imposes a loss of intrinsic properties, therefore not suitable form feature retention objective. 
In Table II, the performance of gamma correction is evaluated with different values of $\gamma$ ranging from 0.25 to 1.25 for each dataset considered in the proposed study. It can be analyzed that the value of PSNR varies depending on the value of $\gamma$ operator. Fig. 4 shows that the graph trend exhibits the consistency of enhancement with the highest PSNR score at a similar range for three datasets. However, one advantage is that the value of $\gamma$ can be adjusted flexibly depending on the requirement of image analysis.

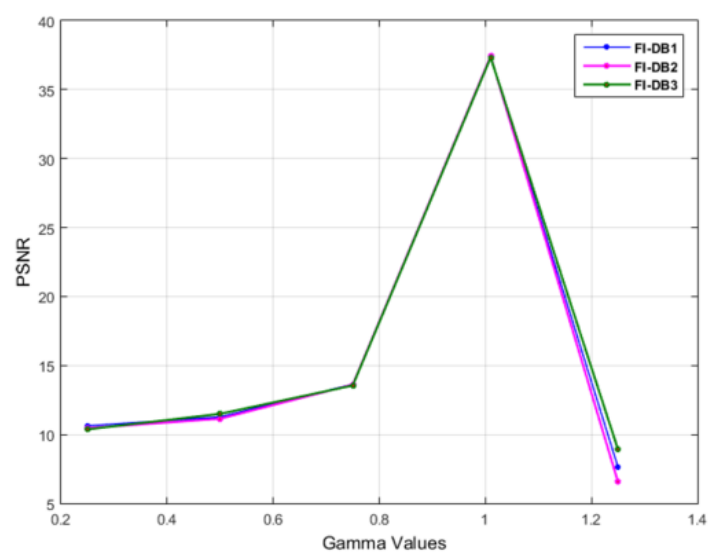

Fig. 4. Observations of the PSRN with Gamma Value Ranging from 0.25 to 1.5 .

\section{E. Equalization of the Histogram}

The typical relationship between the Gray points and their frequency indicates the histogram of the FI defined in equation 6 below:

$\mathrm{FI}(\mathrm{k})=\mathrm{Pn} / \mathrm{N}$

Where $\mathrm{FI}(\mathrm{k})$ is the histogram of FI, where $\mathrm{k}$ indicates the gray points of an FI, Pn is the total number of the pixels in the gray point $\mathrm{k}$, and $\mathrm{N}$ is the summation of all the pixels; therefore, the $\operatorname{FI}(\mathrm{k})$ is the probability distribution of $\mathrm{k}$. The process of the equalization of the histogram adopts a mapping procedure, where each pixel of the input FI is mapped to a new value as $\mathrm{FI}(\mathrm{i}) \rightarrow \mathrm{FI}(\mathrm{j})$ using a mapping function $\mathrm{Mf}(\mathrm{i})$ to get a dynamic range of the output image FI(o) such that:

$\mathrm{FI}(\mathrm{i})=\operatorname{Mf}(\mathrm{i})=(\mathrm{FI}(0)-1) \sum_{k=0}^{i} F I(k)$

Table III demonstrates a quantitative assessment of histogram equalization with different images from the particular datasets, which shows a little better pixel distribution than gamma correction. The quantitative evaluation of the Histogram Equalization technique is performed on various dataset images with their probability distribution of the pixels and the PSNR values. In this method, the larger and smoother areas get over-enhanced, which affects the disappointing appearance. If the fundus images are captured in the low lighting conditions, then the outputs are darker and contain larger smooth regions. Therefore, the histogram equalization process over-enhances the fundus images.

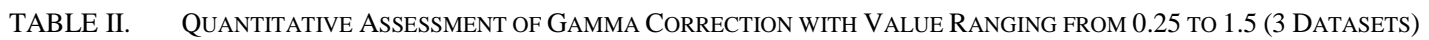

\begin{tabular}{|c|c|c|c|c|c|c|}
\hline \multirow[b]{2}{*}{ DS1 } & Input Image & $\boldsymbol{\gamma}=0.25$ & $\gamma=0.5$ & $\gamma=.75$ & $\gamma=1.01$ & $\gamma=1.25$ \\
\hline & & & & & & \\
\hline \multicolumn{2}{|c|}{ PSNR $=$} & 10.6271 & 11.2672 & 13.6460 & 37.2933 & 7.6411 \\
\hline \multirow[b]{2}{*}{ DS2 } & Input Image & $\gamma=0.25$ & $\gamma=0.5$ & $\gamma=.75$ & $\gamma=1.01$ & $\gamma=1.25$ \\
\hline & & & & & & \\
\hline \multicolumn{2}{|c|}{ PSNR $=$} & 10.4552 & 11.1387 & 13.6083 & 37.4164 & 6.5681 \\
\hline
\end{tabular}

TABLE III. QUANTITATIVE ASSESSMENT OF Histogram EQUALIZATION ON DIFFERENT Fundus IMAGES

\begin{tabular}{|c|c|c|c|}
\hline Dataset & Original Image & Enhanced Image & PSNR \\
\hline DS1 & & & 8.6827 \\
\hline DS2 & & & 10.2113 \\
\hline
\end{tabular}


TABLE IV. QUANTITATIVE ASSESSMENT OF CLAHE ON DIFFERENT FUNDUS IMAGES

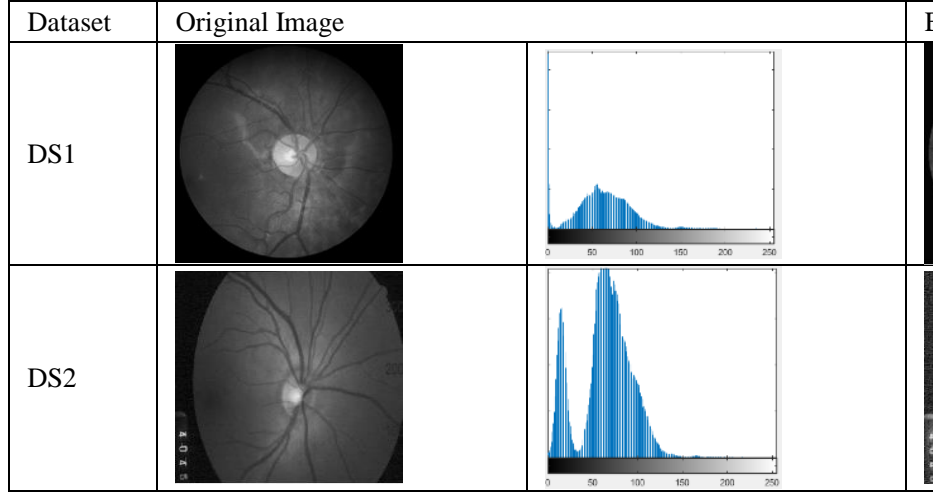

\section{F. Contrast Limited Adaptive Histogram Equalization: CLAHE}

In order to overcome the limitations of the histogram equalization-based enhancement of FI, a method of CLAHE is evaluated, which minimizes the artifacts that resemble noise associated with the uniform regions. The typical process of the CLAHE-for-FI includes the redistribution process of the histogram and intra-block bilinear interpolation. The internal process of the CLAHE-for-FI initially partitions the original FI into a number of the blocks of size $\mathrm{B} \times \mathrm{B}$, and then on each block, the HE-for-FI is implemented. In the process of the HEfor-FI includes three significant operations as \{Hsit, Clipp, Redistribution\}, the specific mapping procedure provides a cumulative distribution from the clipped histogram. Further, the bilinear Interpolation provides the filtered blocks of the noises so that based on the height of the clips the contrast is enhanced. The clip-point $(\mathrm{Cp})$ is computed as in equation 8 as below:

$\mathrm{Cp}=\mathrm{P} / \mathrm{Q}(1+(\mathrm{K} / 100) \mathrm{x} \theta \max )$

Where $\mathrm{P}$ is the pixels in each Block (Bi) of the FI, Q is the dynamic range in the $\mathrm{Bi}, \theta \max$ is the maximum value of the slope, and $\mathrm{K}$ is the clip-factor ranging between [0:100]. The performance evaluation on various dataset images is tabulated in Table IV below with their probability distribution of the pixels and the PSNR values. The block-wise operation used in CLAHE-for-FI provides the enhancements into the contrast in a lower computational complexity. Thus, the operations where the large dataset requires to be fed in the learning models to achieve lower computational complexities during the preprocessing stage of the contrast enhancement, the CLAHEfor-FI is preferable to the HE-for-FI.

\section{G. Retinex}

A Retinex is an image enhancement technique used to adjust contrast and address illumination issues in the image. The algorithm considers logarithmic single-channel intensity images to be processed. The computing steps for Retinex based image enhancement are discussed in algorithm 2 . The explicit function $f_{1}()$ takes the original fundus image $\left(\mathrm{FI}_{\text {ori }}\right)$ that provides the corresponding number of rows(nR) and columns(nC), which indicates the height $(\mathrm{H})$ and width(W), respectively, of the fundus image (FI). The system then initializes a variable $\mathrm{nI}$ as the number of enhanced fundus image iterations. The maximum color value $(\mathrm{Mcv})$ from the

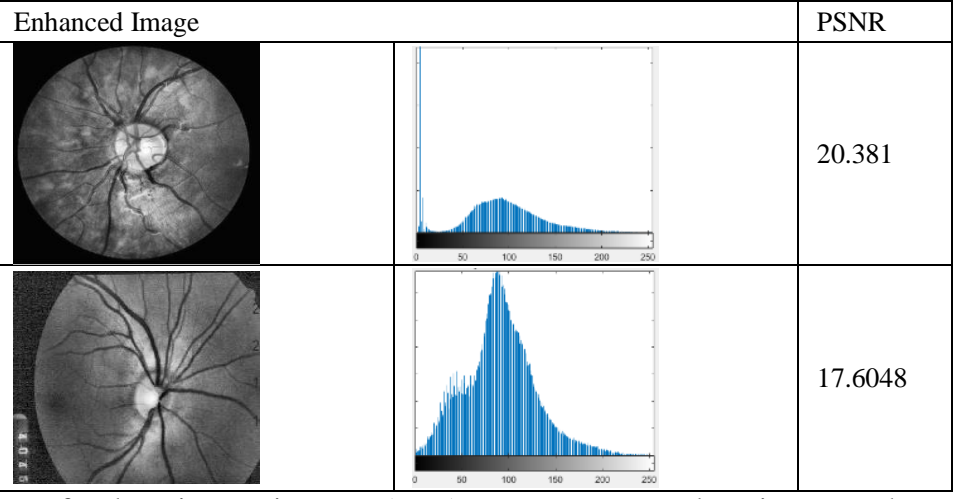

fundus input image $\left(\mathrm{FI}_{\mathrm{ori}}\right)$ gets computed using another explicit function $f_{2}(\mathrm{)}$, which defines the scale of the values possible for the color intensities. Further, an initial shift(s) computation gets performed using equation 9, as shown below.

$S=\sum_{i, j} 2^{\left(f 3\left(\log _{2}\left(f_{\min }(n R, n C)\right)\right)-1\right)}$

Where, $\mathrm{i}=1$ to $\mathrm{nR}$ and $\mathrm{j}=1$ to $\mathrm{nC}$.

Algorithm 2: Retinex based Fundus imageEnhancement

Input: $\mathrm{FI}_{\text {ori }}$

Output: $\mathrm{FI}_{\mathrm{e}}$

Start

1. $[\mathrm{H}, \mathrm{W}]:[\mathrm{nR}, \mathrm{nC}] \leftarrow f l\left(\mathrm{FI}_{\mathrm{ori}}\right)$

2. Initialize $\mathrm{nI}$

3. Compute: Mcv

4. $\mathrm{Mcv} \leftarrow f 2(\mathrm{I})$

5. Perform initial shift: $S \leftarrow \sum_{i, j} 2^{\left(f 3\left(\log _{2}\left(f_{\min }(n R, n C)\right)\right)-1\right)}$

6. $\quad \mathrm{Op}_{[] \mathrm{mxn}} \leftarrow M c v \times \sum_{i, j} n_{(n R, n C)}$

7. Till the condition $|\mathrm{S}|>=1$ )

8. do check: for each nI $\mathrm{Hs} \leftarrow f 4(0, \mathrm{~S})$ Vs $\leftarrow f 4(\mathrm{~S}, 0)$

9. end

10. $S=(-S) / 2$

11. Stop

12. $\mathrm{FI}_{\mathrm{e}} \leftarrow \frac{U O p+O p}{2}$

End

The explicit function $f_{3}()$ is used for truncating the numbers in $\mathrm{nR}$ and $\mathrm{nC}$ to integer, i.e., closer to zero, and the function $f_{\min }()$ is used to compute the smallest element from the $\mathrm{nR}$ and $\mathrm{nC}$. In the next step of the computation, the system performs initialization of the old product (Op) to scale all pixel values of $\mathrm{nR}$, and $\mathrm{nC}$ equal to the value of $\mathrm{Mcv}$, as shown in equation 10.

$O p=M c v \times \sum_{i, j} n_{(n R, n C)}$

Where, $n=1$.

However, the absolute value of $\mathrm{S}$ is greater or equal to 1 than for each nI. In that case, the system performs horizontal (Hs) and vertical shift (Vs) comparison operation using function $f 4()$, with an input argument of shift row (Sr) and 
shift column (Sc). The algorithm initializes the variable UOp as updated Op, which gets computed based on the condition where the sum of the Sr and Sc is greater than zero. The value of $U O p$ is updated with Mcv fas expressed in equation 8.

Once the condition of $(|S|>=1)$ becomes false, then the final enhanced Fundus image $\left(\mathrm{FI}_{\mathrm{e}}\right)$ is obtained as in equation 11 .

$F I_{e}=\frac{U O p+O p}{2}$

The core function of the Retinex algorithm focuses on dynamic ranges and color stability. When capturing an image by fundus imaging devices, it may be possible that certain conditions may cause the image to have a low dynamic range or poor color stability. The color of an object in images remains unchanged under various lighting conditions perceived by HVS, which is called color stability. The key mechanism of Retinex is the estimation of illumination. The Retinex algorithm is based on the path, recursion, and centersurround mechanism to enhance details and textures in the image by illumination removal. Table $\mathrm{V}$ exhibits the qualitative outcome of the preprocessing technique based on the Retinex algorithm for different fundus image samples with their probability distribution of the pixels and the PSNR values.

\section{H. Proposed Image Enhancement}

The fundus images usually suffer from the noises and correct poor luminance due to the absence of an element extent in the picture sensor of the fundus camera and the wrong setting of the lens opening in the camera. The proposed algorithm for fundus image enhancement uses the convolution property in order to mitigate all forms of distortions in the frequency levels. This handles the dark level of the fundus image and adjusts the contrast improved distribution of pixel density within the restricted range. The computing steps for the proposed image enhancement algorithm is discussed in algorithm 3. In the proposed algorithm, the explicit function $f 1()$ takes the original fundus image $\left(\mathrm{FI}_{\text {ori }}\right)$ that gives the corresponding number of rows(nR) and columns(nC), which indicates the height $(\mathrm{H})$ and width $(\mathrm{W})$, of the fundus image (FI). The centroidal components $\mathrm{P}$ and $\mathrm{Q}$ get evaluated as $\mathrm{H} / 2$ and $\mathrm{W} / 2$. The system initializes the maximum high frequency and lowfrequency co-efficient as: $\{\mathrm{rH}, \mathrm{rL}\}$ and the other co-efficient: $\{\mathrm{hm}, \mathrm{d} 0\}$ as per the frequency adjustment requirements of the fundus image contrast.

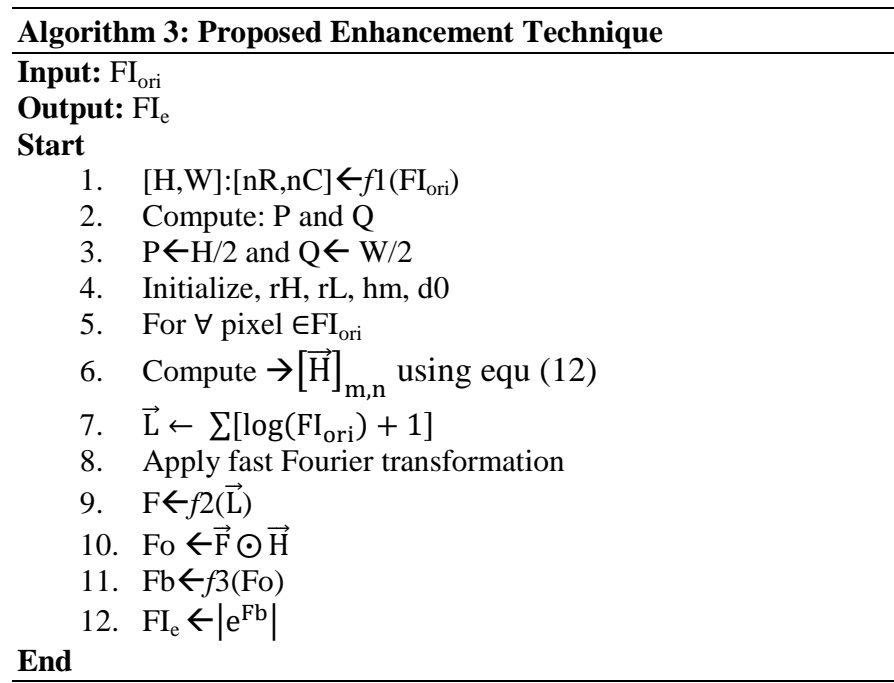

and brightness. Further, for each element of every row, the computation takes place as in equation 12 to update the vector $[\vec{H}]_{m, n}$.

$[\overrightarrow{\mathrm{H}}]_{\mathrm{m}, \mathrm{n}}=(\mathrm{rH}-\mathrm{rL}) \times\left(1-\mathrm{e}^{-h m c}\right)+\mathrm{rL}$

Where, coefficient c can be numerically expressed as follows:

$c=\left(\frac{\left(\sqrt{(i-P / 2)^{2}+(j-Q / 2)^{2}}\right)^{2}}{d 0}\right)$

Where, $\mathrm{i}=1$ to $\mathrm{m}$ and $\mathrm{j}=1$ to $\mathrm{n}$. The low-frequency coefficient (L) computes as in equation 14.

$\overrightarrow{\mathrm{L}}=\sum[\log (\mathrm{I})+1]$

TABLE V. QUANTITATIVE ASSESSMENT OF RETINEX ALGORITHM ON DIFFERENT FuNDUS IMAGES

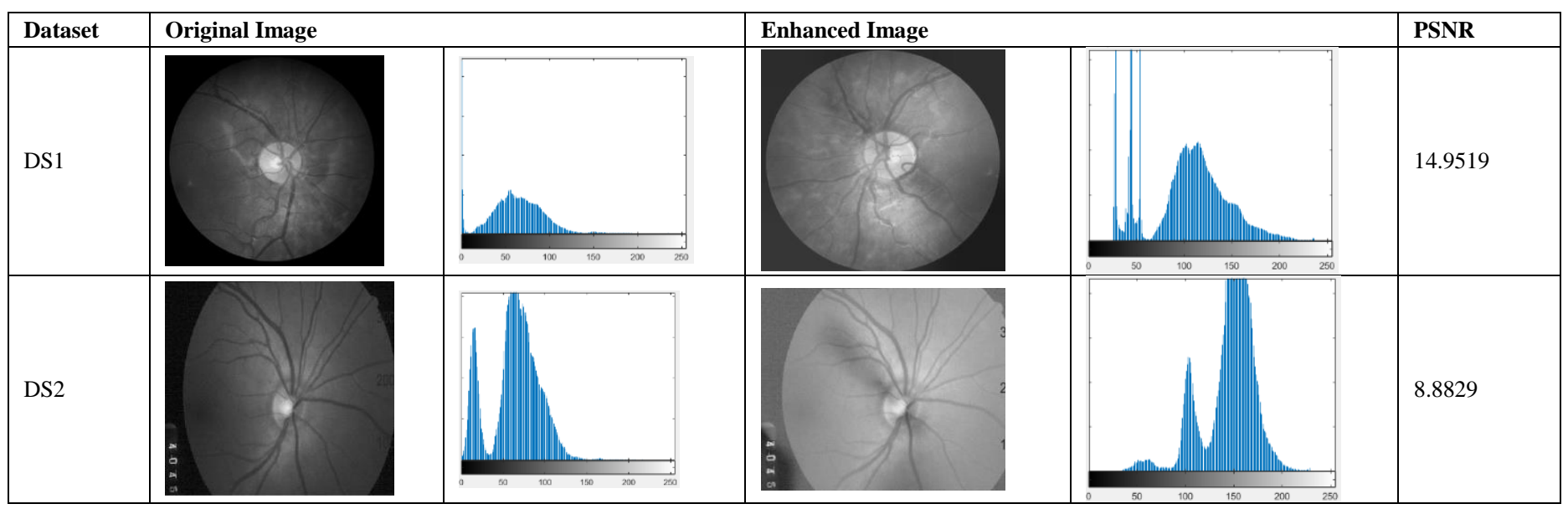


TABLE VI. QuANTITATIVE AsSESSMENT OF THE PROPOSED IMAGE ENHANCEMENT TECHNIQUE ON DIFFERENT FundUS IMAGES

\begin{tabular}{|l|l|l|l|}
\hline Dataset & Original Image & \\
\hline DS1 & & & \\
\hline DS2 & & & \\
\hline & & & \\
\hline & & & \\
\hline
\end{tabular}

In the next step,an explicit function $f 2()$ is used for twodimensional fast Fourier transformation(2D-FFT) that takes $\vec{L}$ to provide the corresponding co-efficient as $\vec{F}$. Many of the border frequency $(\mathrm{Fb})$ components are not centered, which gets centered by using explicit function $f 3()$ for applying inverse $2 \mathrm{D}$-FFT on filout portions (Fo). The value of filout is the vector product of $\vec{F}$ and $\vec{H}$. The final enhanced fundus image $\left(\mathrm{FI}_{\mathrm{e}}\right)$ yields better visual perception as per the coefficient adjustments with the operations, as in equation 7.

$\mathrm{FI}_{\mathrm{e}}=\operatorname{Abs}\left(\mathrm{e}^{\mathrm{Fb}}\right)$

In Table VI, a quantitative analysis of the proposed image enhancement algorithm for different fundus image samples is presented. The fundus image enhancement using a proposed method is produced based on the convolution operation linear combinations of the neighboring input image pixels.

\section{RESULT ANALYSIS}

This section discusses the outcomes and performance analysis of the proposed framework concerning PSNR and SSIM quality metrics. The implementation and design of the proposed framework are carried out on a numerical computing tool MatLab. A detailed description of the performance metrics for evaluating existing techniques and proposed techniques for fundus image preprocessing is discussed as follows:

\section{A. PSNR (Peak Signal to Noise Ratio)}

This metric computes the value in decibels $(\mathrm{dB})$ between the original and enhanced fundus images as a visual quality measurement. The higher value of PSNR indicates a better visual perception. There exists a fundamental relationship between the error and PSNR. Whenever the fundus image

\begin{tabular}{|l|l|l|}
\hline Enhanced Image & PSNR \\
\hline & & 23.3258 \\
\hline & & \\
\hline & & \\
\hline
\end{tabular}

undergoes a treatment of enhancement while reconstructing the image, and if there are some losses, it is measured by mean square error (MSE), and the peak error is mapped with PSNR. The value of PSNR is as in equation 17, which uses MSE as in equation 16.

$$
\begin{aligned}
& \text { MSE }=\frac{\sum_{\mathrm{m}, \mathrm{n}}\left[\mathrm{I}_{\text {org }}-\mathrm{I}_{\mathrm{enc}}\right]^{2}}{\mathrm{~m} \times \mathrm{n}} \\
& \mathrm{PSNR}=10 \log _{10}\left(\frac{\mathrm{R}^{2}}{\mathrm{MSE}}\right)
\end{aligned}
$$

Where $\mathrm{R}$ is the maximum variation in the $\mathrm{I}_{\text {org }}$ for doubleprecision, it is ' 1 ', and for 8-bit unsigned integer: 255.

\section{B. SSIM (Structural Similarity Index)}

SSIM represents a human visual system-oriented image quality metric that deals with the similarity between the input and output images. The computation of SSIM is carried out over multiple windows of an image as numerically represented as follows:

$\operatorname{SSIM}(x, y)=\frac{\left(2 \mu_{x} \mu_{y}+c 1\right)\left(2 \sigma_{x y}+c 2\right)}{\left(\mu_{x}^{2}+\mu_{y}^{2}+c 1\right)\left(\sigma_{x}^{2}+\sigma_{y}^{2}+c 2\right)}$

Where, $\mu_{x}$ and $\mu_{y}$ both refer to the mean value of $\mathrm{x}$ and $\mathrm{y}$, respectively, $\sigma_{x}^{2}$ and $\sigma_{y}^{2}$ denotes variance value of the $\mathrm{x}$ and $\mathrm{y}$, respectively. $\sigma_{x y}$ indicates covariance. The $c 1$ and $c 2$ represent constraints whose values lie in the range of pixel value 255 .

1) Visual outcome analysis: This section presents a qualitative analysis to visualize the quality of the enhanced images obtained from implemented existing and proposed enhancement techniques. The visual outcome obtained from implemented techniques over two different images from both datasets is shown in Table VII.

\begin{tabular}{|c|c|c|c|c|c|c|c|}
\hline & Input image & GrayScale & $\begin{array}{l}\text { Gamma } \\
\text { correction }\end{array}$ & Histogram & CLAHE & Retinex & Proposed \\
\hline $\begin{array}{l}\text { CHASEDB1 } \\
\text { (Image_08R) }\end{array}$ & 8 & & & & & 8 & \\
\hline $\begin{array}{l}\text { DRIONS_DB } \\
\text { (Image 068) }\end{array}$ & & & & & & & \\
\hline
\end{tabular}

TABLE VII. VISUAL OUTCOME OF DIFFERENT TECHNIQUES FOR THREE DIFFERENT FundUS IMAGES 
In Table VI, qualitative analysis is shown from the perspective of HVS for each technique over two fundus images considered from different datasets. It can be clearly analyzed that the proposed image enhancement techniques provide a clear view of the enhanced fundus image compared to other techniques. In the case of gamma correction, the evaluation is carried out considering the fixed value of the gamma operator for each performance evaluation instance. The visual outcome exhibits that gamma-corrected images suffer from poor brightness. However, the performance of gamma correction largely depends on the gamma operator value. The adjustment of brightness tone can be adjusted by varying the value of the gamma operator between $[0,1]$. In the case of the histogram, the enhanced images are subjected to over brightness issue. The main disadvantage of histogrambased image enhancement is that the image is usually prone to over-amplification in comparatively uniform regions of an image. However, this limitation of the histogram is overcome by CLAHE as it limits the over-enhanced factor of the image. The CLAHE is mostly an adopted technique for image enhancement compared to the adaptive histogram technique. Also, the Retinex techniques do not provide better visual quality of the image, and the performance varies in each test case of the images. The comparative analysis based on quantitative assessment for each implemented technique in terms of PSNR and SSIM is shown in Table VIII.

2) Numerical outcome analysis: This section presents a quantitative analysis based on the numerical outcome concerning PSNR and SSIM value of input fundus images processed by implemented preprocessing techniques. The quantified value obtained for the enhanced output images is given in Table VIII as follows.

In Table VIII, the numerical analysis is shown for 20 fundus images taken from individual datasets for the performance evaluation of the proposed enhancement technique and existing technique. Therefore, a total of 40 fundus images, i.e., 20 fundus images, are taken from the CHASEDB1 dataset, and 20 fundus images from the DRIONS_DB dataset are enhanced using implemented techniques, including existing and proposed enhancement methods. The Numerical output is computed based on the mean value of PSNR for 20 fundus images processed via each individual preprocessing technique under consideration of the proposed work. Fig. 5 demonstrates the comparative analysis for assessing the effectiveness of the proposed system with the existing system based on the mean of PNSR score.

TABLE VIII. NUMERICAL OUTCOME

\begin{tabular}{|l|l|l|l|l|}
\hline \multirow{2}{*}{ TECHNIQUES } & \multicolumn{2}{|l|}{$\begin{array}{l}\text { Dataset: } \\
\text { CHASEDB1(DS1) }\end{array}$} & \multicolumn{2}{l|}{$\begin{array}{l}\text { Dataset: DRIONS_DB } \\
\text { (DS2) }\end{array}$} \\
\cline { 2 - 5 } & PSNR & SSIM & PSNR & SSIM \\
\hline GC & 13.7523 & 0.7684 & 15.0515 & 0.7150 \\
\hline AHE & 9.9658 & 0.4869 & 10.0935 & 0.5687 \\
\hline CLAHE & 20.8603 & 0.6178 & 18.4775 & 0.6569 \\
\hline RETINEX & 14.1990 & 0.6186 & 10.1988 & 0.6631 \\
\hline PROPOSED & 23.7442 & 0.9623 & 25.0495 & 0.8275 \\
\hline
\end{tabular}

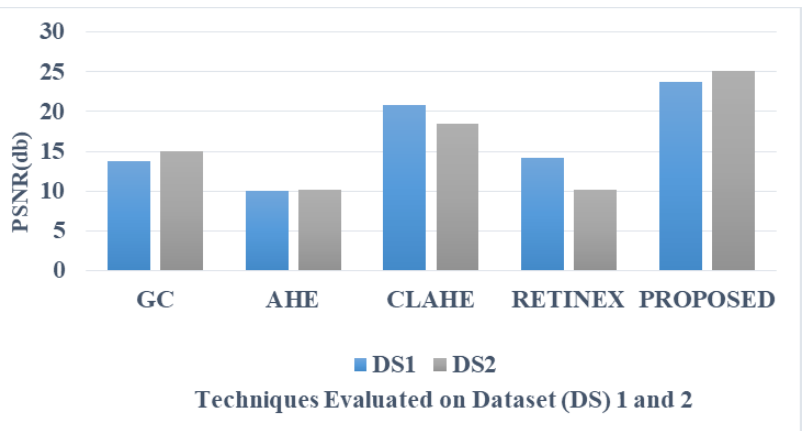

Fig. 5. Comparative Performance for different Preprocessing Techniques in Terms of PSNR.

From the comparative analysis in Fig. 5, it can be seen that the proposed image enhancement algorithm outperforms the other enhancement techniques regarding PSNR. However, after the proposed system, the CLAHE technique has maintained a good score of PNSR for different images compared to other existing techniques. In Fig. 6, a comparative analysis is presented based on the mean SSIM value obtained for each implemented technique. Based on the analysis, the proposed image enhancement technique outperforms the other enhancement techniques regarding SSIM. However, gamma correction has maintained a better mean SSIM for different images after the proposed system. It has been analyzed from both analysis that each method has a different performance score.

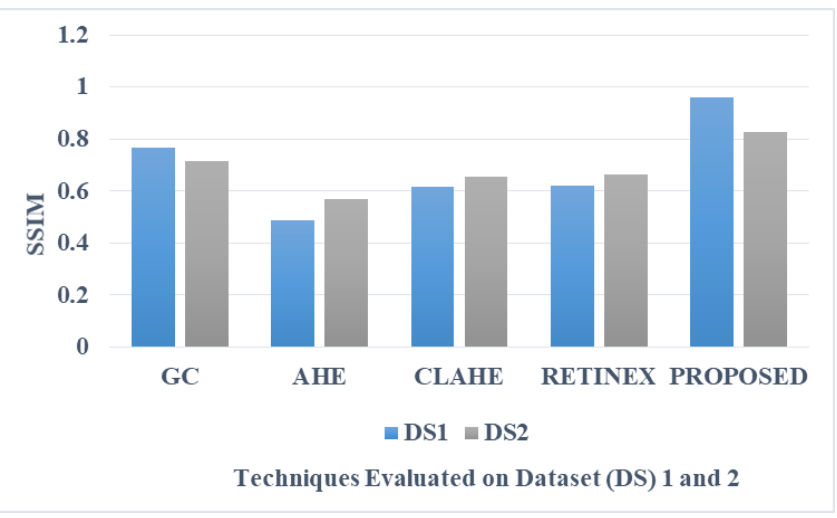

Fig. 6. Comparative Performance for different Preprocessing Techniques in Terms of SSIM.

The proposed enhancement technique has provided a better visual quality in the enhanced image. In the case of gamma correction, the PSNR and SSIM value is comparatively low compared to the proposed technique. The main disadvantage of gamma correction is that it has restricted precision, and multiple input values may be usually mapped to the same output or vice-versa. It applies adjustment of brightness on overall images. Hence, color space is also not perceptually evenly distributed.

Similarly, it has been analyzed that the adaptive histogram is also not suitable for fundus image enhancement. The histogram equalization algorithms provided unsatisfactory outcomes while dealing with fundus images obtained from a different dataset. This technique is associated with overamplification of brightness and non-uniform distribution of 
illumination images. It is because the histogram redistribution leads to unfitting pixel values in certain regions of the fundus image. The image processed with CLAHE yields a better outcome than the ordinary histogram equalization, gamma correction, and Retinex techniques. The CLAHE overcomes over-amplification in brightness as it operates on multiple fixed regions of the image known as tiles, in contrast to the overall image. However, CLAHE has certain limitations that, for certain images, it introduces noise application in flat tiles. CLAHE is computationally expensive compared to the ordinary histogram equalization technique and proposed technique. However, CLAHE outperforms other existing techniques in terms of PSNR and SSIM. But less effective compared to gamma correction in terms of SSIM. The Retinex technique is widely used in the literature to deal with poor illumination in the image. It has been analyzed that the performance of the Retinex technique quite varies for different images as its performance depends on the visual feature of images. It cannot be suitable for all types of fundus images. In Retinex based enhancements, reflectance is calculated as the image ratio to the smoothed version of the image, which is considered an approximation of illumination. The disadvantage of the retinex technique is that it can simultaneously offer dynamic compressions and visual tone reproduction. Also, the input image processed by retinex is prone to color distortion, loss of details due to halo artifacts in the output image. Another significant issue is observed while its execution is that it takes a long run time to process the image due to the involvement of the iterative process.

The proposed enhancement technique is quite flexible and evaluated with a large fundus image dataset and has a faster response time to execute preprocessing operation over input fundus image. The proposed enhancement technique takes very little run time as it adopts a simple implementation conceptually to process the input image in the frequency domain by constructing a filter vector based on different frequency coefficients and centroidal image components. On the other hand, the convolution operation in the image processing leads to yielding a better form of output enhanced fundus image. Remarkably, the proposed enhancement technique smoothens, sharpens the edges, and preserves the useful information in the output image. Based on the outcome obtained and comparative analysis, the proposed enhancement technique can be claimed to be efficient and suitable to operate on fundus images to draw a significant clinical conclusion towards eye-related disease detection.

\section{CONCLUSION}

Medical imaging introduces a high degree of vision into the healthcare system, leading to widespread changes in the diagnosis and treatment of Glaucoma and diabetic retinopathy. The presence of noise, less contrast factor, and the reduction in fundus images' sharpness bring vagueness in the clinical analysis. This paper proposes an adaptive framework to carry out comprehensive image enhancement over fundus images. A user-friendly and interactive framework is introduced in the proposed study that allows users to select a desirable technique to address different issue-related images and meet preprocessing needs. The integration of the interpolation technique in the proposed framework allows the user or ophthalmologist to deal with an imbalanced dataset consisting of variable sizes of images. The integration of the RoI extraction technique into the proposed framework is also an essential contribution, which will assist ophthalmologists in carrying out analysis on the interest area of the Fundus. Since the performance of each technique also depends on the visual characteristics of the input image. The selection of the enhancement techniques can be made based on performance metrics. In certain circumstances, multiple techniques can be checked with the input image, where the suitability of the selected technique is based on the output image quality metrics, i.e., PSNR and SSIM. Therefore, the proposed work contributes to providing deep analysis and understanding of the eye-related disease to provide better treatment. The study aimed to offer a flexible approach to diagnose the Fundus image deeply without compromising any performance-related issue. However, the scope of this framework is not only limited to the domain of fundus image enhancement. The uniqueness of the proposed framework is that it is adaptive and can be efficiently applied to other medical imaging domains, as it has layers of image enhancements techniques. In the future work, the enhanced image obtained from the proposed framework will be used to perform segmentation and glaucoma classification using machine learning technique.

\section{REFERENCES}

[1] X. Gu, Y. Wong, L. Shou, P. Peng, G. Chen, and M. S. Kankanhalli, "Multi-Modal and Multidomain Embedding Learning for Fashion Retrieval and Analysis," in IEEE Transactions on Multimedia, vol. 21, no. 6, pp. 1524-1537, June 2019.

[2] Alexander A, Jiang A, Ferreira C, Zurkiya D. An intelligent future for medical imaging: a market outlook on artificial intelligence for medical imaging. Journal of the American College of Radiology. 2020 Jan 1;17(1):165-70.

[3] https://www.openpr.com/news/1786173/global-ai-medical-imagingmarket-to-grow-at-usd-264-85-billion-by-2026.html.

[4] M. C. V. Stella Mary, E. B. Rajsingh and G. R. Naik, "Retinal Fundus Image Analysis for Diagnosis of Glaucoma: A Comprehensive Survey," in IEEE Access, vol. 4, pp. 4327-4354, 2016,

[5] Chalakkala RJ, Abdullaa WH, Hongb SC. Fundus retinal image analyses for screening and diagnosing diabetic retinopathy, macular edema, and glaucoma disorders. Diabetes and Fundus OCT. 2020 Apr 15:59.

[6] Lapierre-Landry M, Carroll J, Skala MC. Imaging retinal melanin: a review of current technologies. Journal of biological engineering. 2018 Dec 1;12(1):29.

[7] Sudeshna Sil Kar and Santi P. Maity, 'Automatic Detection of Retinal Lesions for Screening of Diabetic Retinopathy', IEEE Transactions on Biomedical Engineering, Vol.65, No.3, pp.608-618, March-2018.

[8] Sangita Bharkad, 'Automatic Segmentation of Optic Disc in Retinal Images', Biomedical SignalProcessing and control, Vol.31, pp.483-498, 2017.

[9] Jebaseeli, T. Jemima, C. Anand Deva Durai, and J. Dinesh Peter, 'Segmentation of Type II Diabetic Patient's Retinal Blood Vessel to Diagnose Diabetic Retinopathy In Computer Aided Intervention and Diagnostics in Clinical and Medical Images', pp. 153-160. Springer, Cham, 2019.

[10] Bhat, Shreenidhi H., and Preetham Kumar, 'Segmentation of Optic Disc by Localized Active Contour Model in Retinal Fundus Image', Smart Innovations in Communication and Computational Sciences, pp. 35-44, Springer, Singapore, 2019.

[11] Sahu S, Singh AK, Ghrera SP, Elhoseny M. An approach for denoising and contrast enhancement of retinal fundus image using CLAHE. Optics \& Laser Technology. 2019 Feb 1; 110:87-98.

[12] H. Ab Rahim, A.S. Ibrahim, WMDW Zaki, A. Hussain, Methods to enhance digital fundus image for diabetic retinopathy detection, in: 2014 
IEEE 10th International Colloquium on Signal Processing \& its Applications (CSPA), IEEE, 2014, pp. 221-224.

[13] Y. Elloumi, M. Akil, N. Kehtarnavaz, A computationally efficient retina detection and enhancement image processing pipeline for smartphonecaptured fundus images, J. Multimedia Inf. Syst. (2018).

[14] Anilet Bala A, Aruna Priya P, Maik V. Retinal image enhancement using adaptive histogram equalization tuned with nonsimilar grouping curvelet. International Journal of Imaging Systems and Technology.

[15] Hari VS, Raj VJ, Gopikakumari R. Quadratic filter for the enhancement of edges in retinal images for the efficient detection and localization of diabetic retinopathy. Pattern Analysis and Applications. 2017 Feb 1;20(1):145-65.

[16] Abdallah MB, Malek J, Azar AT, Belmabrouk H, Monreal JE. Performance evaluation of several anisotropic diffusion filters for fundus imaging. International Journal of Intelligent Engineering Informatics. 2015;3(1):66-90.

[17] Vinodhini CA, Sabena S, Ramesh LS. A Robust and Fast Fundus Image Enhancement by Dehazing. InInternational Conference On Computational Vision and Bio Inspired Computing 2018 Nov 29 (pp. 1111-1119). Springer, Cham.

[18] Bhardwaj C, Jain S, Sood M. Automated optical disc segmentation and blood vessel extraction for fundus images using ophthalmic image processing. InInternational Conference on Advanced Informatics for Computing Research 2018 Jul 14 (pp. 182-194). Springer, Singapore.

[19] Hassan G, Hassanien AE. Retinal fundus vasculature multilevel segmentation using whale optimization algorithm. Signal, Image and Video Processing. 2018 Feb 1;12(2):263-70.

[20] Sadia H, Azeem F, Ullah H, Mahmood Z, Khattak S, Khan GZ. Color Image Enhancement Using Multiscale Retinex with Guided Filter.
In2018 International Conference on Frontiers of Information Technology (FIT) 2018 Dec 17 (pp. 82-87). IEEE.

[21] Mahmood Z, Muhammad N, Bibi N, Malik YM, Ahmed N. Human visual enhancement using Multi Scale Retinex. Informatics in Medicine Unlocked. 2018 Jan 1;13:9-20.

[22] Sathananthavathi, V., Indumathi, G. Particle Swarm Optimization Based Retinal Image Enhancement. Wireless Pers Commun (2021).

[23] Alwazzan, M.J., Ismael, M.A. \& Ahmed, A.N. A Hybrid Algorithm to Enhance Colour Retinal Fundus Images Using a Wiener Filter and CLAHE. J Digit Imaging (2021).

[24] Bataineh, B., Almotairi, K.H. Enhancement Method for Color Retinal Fundus Images Based on Structural Details and Illumination Improvements. Arab J Sci Eng (2021).

[25] Carrillo, L. Bautista, J. Villamizar, J. Rueda, M. Sanchez and D. rueda, "Glaucoma Detection Using Fundus Images of The Eye," 2019 XXII Symposium on Image, Signal Processing and Artificial Vision (STSIVA), Bucaramanga, Colombia, 2019, pp. 1-4,

[26] A. Raj, A. K. Tiwari and M. G. Martini, "Fundus image quality assessment: survey, challenges, and future scope," in IET Image Processing, vol. 13, no. 8, pp. 1211-1224, 206 2019, doi: 10.1049/ietipr.2018.6212.

[27] Mittal, K., Rajam, V.M.A. Computerized retinal image analysis - a survey. Multimed Tools Appl 79, 22389-22421 (2020).

[28] Juneja, M., Thakur, S., Wani, A. et al. DC-Gnet for detection of Glaucoma in retinal fundus imaging. Machine Vision and Applications 31, 34 (2020).

[29] Gómez-Valverde JJ, Antón A, Fatti G, Liefers B, Herranz A, Santos A, Sánchez CI, Ledesma-Carbayo MJ. Automatic glaucoma classification using color fundus images based on convolutional neural networks and transfer learning. Biomedical optics express. 2019 Feb 1;10(2):892-913. 